\title{
A Formative Evaluation of Zimbabwe's HIV Testing Services Strategy, 2019 - A Cross-sectional Study
}

\author{
Mugauri HD ${ }^{1^{*}}$, Ncube $\mathrm{G}^{1}$, Chikondowa $\mathrm{I}^{1}$, Dupwa $\mathrm{B}^{1}$, Gwashure $\mathrm{S}^{1}$, Nyazema $\mathrm{L}^{1}$, Mugurungi $\mathrm{O}^{1}$, \\ Chiwungwe $\mathrm{F}^{2}$ and Rwodzi $\mathrm{D}^{2}$
}

${ }^{1}$ Ministry of Health and Child Care, AIDS and TB Unit, Harare, Zimbabwe

${ }^{2}$ Clinton Health Access Initiative, Harare, Zimbabwe

*Corresponding author: Mugauri HD, Ministry of Health and Child Care, AIDS and TB Unit, ${ }^{\text {nd }}$ Floor Mukwati Building, Corner Livingstone and Five Avenue, Harare, Zimbabwe, Tel: +263772314894;

E-mail: dumiwaboka@gmail.com

Citation: Mugauri HD, Ncube G, Chikondowa I, Dupwa B, Gwashure S, et al. (2020) A Formative Evaluation of Zimbabwe's HIV Testing Services Strategy, 2019 - A Cross-sectional Study. J AIDS Immune Syst 1: 103

Article history: Received: 17 September 2020, Accepted: 24 September 2020, Published: 12 October 2020

\section{Abstract}

Aim: We evaluated the HIV Testing Services (HTS) Strategy (2017-2020) at mid-term to ascertain adherence to implementation protocol and recommend critical adjustments. Zimbabwe recorded an exponential increase in HIV tests, from 1.7 million (2011) to 3 million (2018) against the declining yield of $20 \%(327,000)$ to $6 \%$ (174,000 tests) respectively. Cost-effective HIV testing requires targeted strategies to identify $95 \%$ of people living with HIV in Zimbabwe by 2020. HIV testing is the entry point to HIV prevention and treatment services.

Subject and Methods: We conducted a cross-sectional study of 36 selected implementing sites. Data were collected through structured preforms from clinical records and key informant interviews were conducted among health care workers across all levels of service provision. Clinical documentation data were triangulated with the routinely collected electronic database. Quantitative data were summarized as proportions, odds ratios and adjusted odds ratios at $5 \%$ significance level.

Results: Of 34,605 HIV tests done, females constituted 70\% ( $\mathrm{n}=24,221)$. An 11.1\% discrepancy between abstracted data from registers $(34,605)$ and electronic databases $(31,171)$ was observed. Provider-initiated testing accounted for $25.8 \%(n=8,938)$, whilst $67.2 \%(n=23,265)$ were retests. Positivity was highest among 25-49 age category (aOR=2.77 [95\% CI, 1.97-3.9]), whilst males had 20\% more chances of testing HIV positive (95\% CI, 1.09-1.33). Index contacts were 5 times more likely to test HIV positive (95\%CI, 2.68-9.19), whilst the chances of testing positive at first test was 56\% (95\%CI, 1.41-1.71) in adjusted analysis. Cascades indicated 6.9\% ( $\mathrm{n}=326)$ attrition from diagnosis to treatment. Among 1,233 self-testers, 2.9\% $(\mathrm{n}=35)$ reported reactive results, $80 \%(\mathrm{n}=28)$ were confirmed through provider testing.

Conclusion: Targeted HIV testing through screening eligibility for testing and vigilant index contact eliciting, tracing and testing may improve yield and efficiency, reducing the number of kits needed to identify positives. Strengthening this approach will facilitate HTS target achievement and HIV epidemic control.

Keywords: Targeted Testing; HIV Testing Services; Index Contact Tracing; HIV Testing Cascades; HIV Self-Testing; HIV Epidemiology

\section{Introduction}

Zimbabwe's HIV prevalence is on a descending trajectory, from 29\% in 1999 to an encouraging $14 \%$ in 2017 [1]. Key to the decreasing prevalence is the notable reduction in new infections, which dropped to 41,000 in 2017, from 79,000 in 2010, in a country with 75\% antiretroviral therapy (ART) access and markedly decreased mortality [2]. Decreasing new infections, against population growth of $2.3 \%$ has been largely attributable to an intervention mix that hinges on structured HIV Testing Services (HTS), suggesting that achieving epidemic control is on course [3]. Testing for HIV is the entry point to a result informed HIV prevention, treatment, care and support package.

However, HIV testing yield has also decreased from $20 \%$ in 2011, to $6 \%$ in 2018, against consistently surpassed HIV testing targets of between 10-15\%, raising concern over efficient utilisation of the testing resources (Figure 1). 


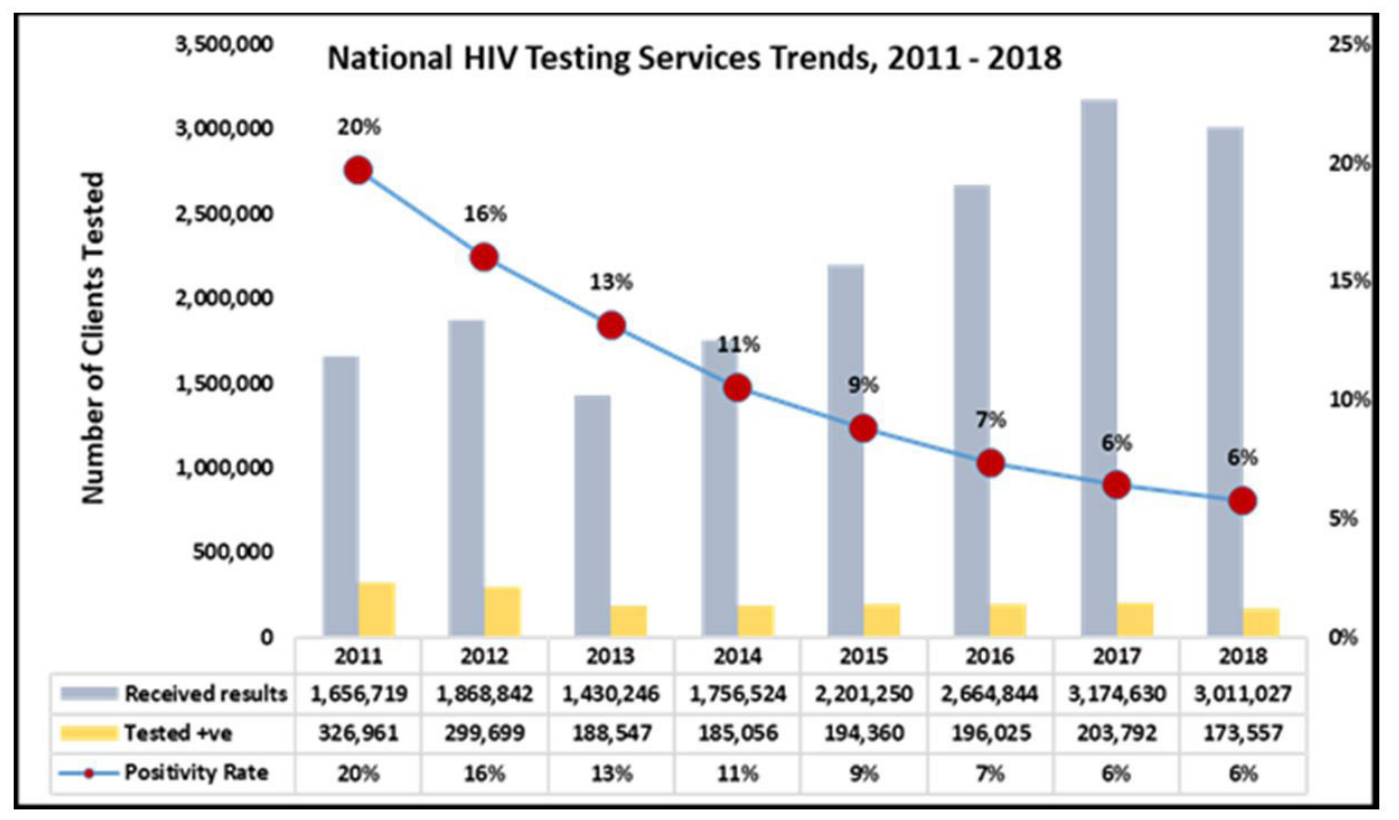

Figure 1: National HIV Testing Services Trends, 2011- 2018

In 2013, Zimbabwe's Ministry of Health and Child Care (MOHCC) launched the 2013-2015 HIV Testing and Counselling Strategy which detailed a roadmap to increase coverage and access to testing services for the general population and ensure that $85 \%$ of men and women know their status and receive a standard package of HIV testing and counselling services [4]. This resulted in an increase in the number of health facilities offering Provider-Initiated Testing and Counselling (PITC) from $80 \%$ to $95 \%$ by 2015 , coupled with innovative strategies of conducting HTS campaigns to increase access to testing services and linkages to prevention treatment care and support [4]. A review of this strategy gave birth to the current HTS, 2017-2020 which strategizes how the 90-90-90 UNAIDS Global Fast Track targets will be achieved as a critical milestone to ending AIDS as a public health threat by 2030 [5,6].

Equestrian to the critical lessons learnt from the previous strategy, the current HTS Strategy (2017-2020) endeavours to refocus the critical service to achieve epidemic control. Knowledge of one's HIV status has been identified as one of the critical cues to action in reducing new infections through an effective linkage package that are summarised in risk reduction and personal behaviour modification, among others [7]. In this new strategy, a target shift from testing for coverage to targeted testing is emphasized, which has demonstrated to increase yield, against reduced testing numbers through innovative strategies such as HIV self-testing, facilityand community-based index client testing and targeted testing of high-risk populations $[2,8]$.

This strategy endeavors to strengthen program implementation through comprehensive approaches across six thematic areas namely: (i) management and coordination, (ii) service delivery, (iii) demand generation, (iv) strategic information, (v) supply chain management and (vi) quality assurance. It is critical, before summative evaluation, to assess the effectiveness of the current strategy, and make necessary adjustments, if needed, and ensure that the overall goal is achieved at the end, which was the main objective of this evaluation. Although aggregate HIV testing figures from 2017 suggested that the annual targets may have been met, the absence of mechanisms or data at the national and sub-national level to determine if this performance was a result of strategy implementation adds to the rationale to conduct a mid-term evaluation of the strategy [9].

Furthermore, there is limited information on how the national HTS strategy and guidelines have been translated into implementation at different facility levels and the utilized testing models in use. We, therefore, sort to evaluate, at mid-implementation, the progress made to date, towards achieving the set objective with, and specifically to;

- Determine the testing models currently being implemented in health facilities and their corresponding yields.

- Evaluate health care workers knowledge, attitudes and practices regarding the implementation of the HTS strategy.

- Assess the comprehensiveness of the pre and post-test counselling messages delivered to clients.

- Identify the challenges and opportunities that exist in the monitoring and evaluation of HIV testing services.

- Recommend the ideal mix of HTS models to ensure achievement of set strategic goals.

\section{Materials and Methods}

\section{Study Design}

We conducted a cross-sectional study, with an analytical component.

\section{Setting}

General Setting: Zimbabwe is a landlocked, low-income country in Southern Africa located between Botswana, South Africa, Mozambique and Zambia with an estimated population of 16 million and human development index of 0.516 , ranked number 154 globally out of 189 
countries in 2016 [5]. The country is divided into two urban provinces, eight rural provinces and 62 districts. The capital city is Harare and other major cities include Bulawayo, Gweru, Kadoma, Kwekwe, Masvingo and Mutare [3].

Zimbabwe National HIV Programme: The AIDS and TB Programme (ATP) coordinates the development of HIV/AIDS health policies and set up of national standards and guidelines as part of the national response to HIV in Zimbabwe. Four sub-units under ATP, namely; HIV Prevention, Care and Treatment, Prevention of Mother to Child Transmission (PMTCT) and Monitoring and Evaluation ( $\mathrm{M} \& \mathrm{E})$, are mandated to ensure seamless yet specialised programming to ensure adequate response to the pandemic [10].

Study Site: The study sites were four provinces, selected out of 10 provinces in Zimbabwe. Manicaland is a province in eastern Zimbabwe. After Harare Province, it is the country's second-most populous province, with a population of 1.75 million, as of the 2012 census [11]. The province is divided into ten administrative subdivisions of seven rural districts and three towns/councils, including the provincial capital, Mutare. Bordered by Mashonaland East Province to the northwest, Midlands Province to the west, Masvingo Province to the southwest, and Mozambique to the east, Manicaland covers an area of 36,459 square kilometres (14,077 sq $\mathrm{mi}$ ), equal to $9.28 \%$ of the total area of Zimbabwe.

Its economy is largely centred around industry and agriculture, particularly manufacturing, diamond and gold mining, timber, tea and coffee plantations, and tourism [5]. Mashonaland West Province Mashonaland West is located to the North of Zimbabwe and shares the international border with Zambia in the North. Internally, the borders of the province with Midlands Province in the West, Matabeleland North in the West, Mashonaland Central in the East, Harare and Mashonaland East in South East. Sitting on an area of 57,441 square kilometres, with four distinct agro-ecological regions, the Province is home to a population of over 1.5 million people (World Population Review 2018). The people are united by their rich culture with the mainstay of their livelihoods being derived from natural endowments which include minerals, water bodies, wildlife, rich biodiversity and vast arable land. has its administrative capital in Chinhoyi. Chinhoyi is the capital of the province.

Matabeleland South Province covers the south-eastern plateau of Zimbabwe and it stretches to the Botswana border on the east and borders South Africa on the South. It is one of Zimbabwe's ten provinces and its administrative city if Lupane.

Matabeleland South is a province in southwestern Zimbabwe. With a population of 683,893 as of the 2012 census, it is the country's least populous province. After Matabeleland North, it is Zimbabwe's second-least densely populated province. The province is divided into six districts. Gwanda is the capital, and the Beitbridge is the province's largest town [11]. Midlands province has an area of 49,166 square kilometres and a population of 1,614,941 (2012). Gweru is the capital of the province. As a central point in Zimbabwe, it has a blend of all major languages in Zimbabwe, including Shona, Ndebele, Tswana, Suthu, Chewa among various other languages spoken in Zimbabwe [12].

\section{Client Population}

All clients who were tested for HIV and documented in HIV Testing registers, including self-tests, in the 37 sampled facilities between 1 October 2018 to 31 December 2018 were included in the survey.

\section{Sampling}

Purposive sampling criteria were utilised to select 4 out of 10 Zimbabwe provinces, from which 3-4 facilities each were purposively selected (Figure 2).

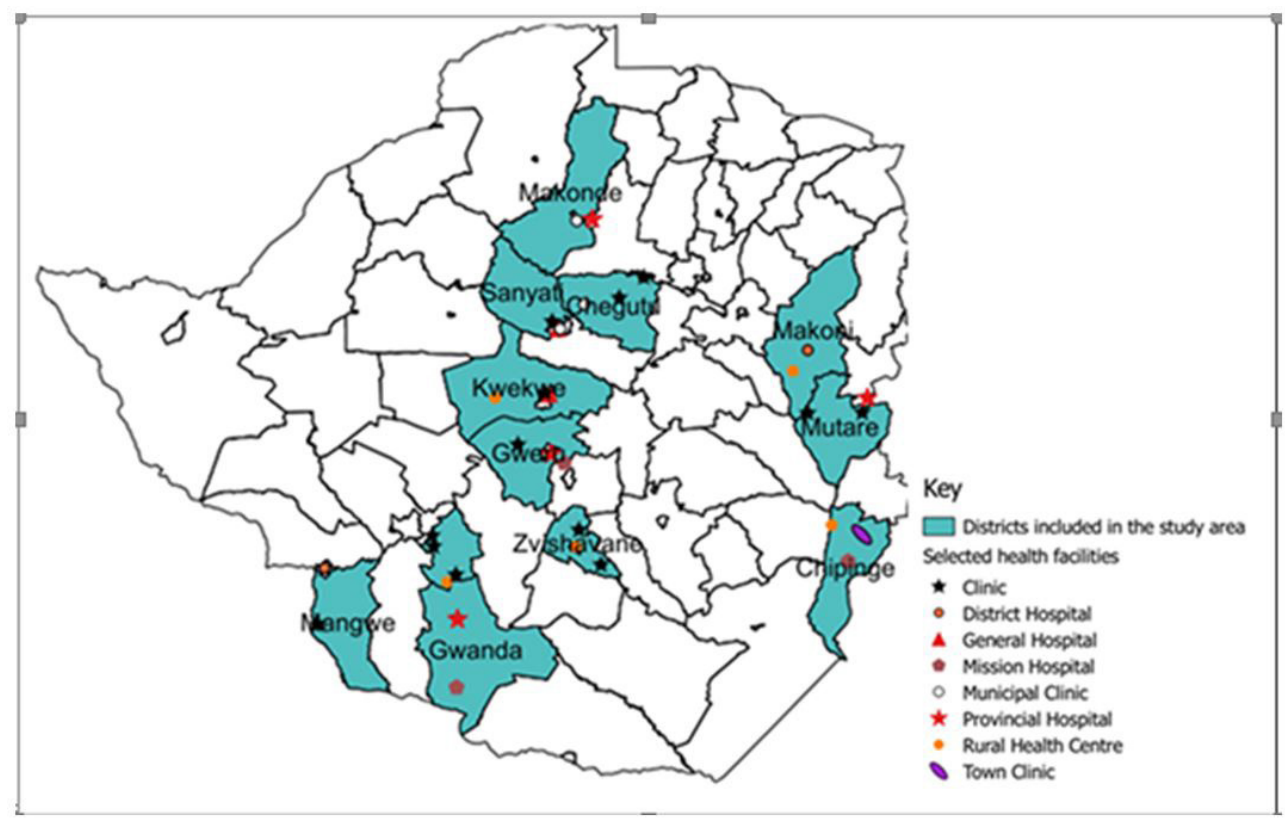

Figure 2: Mapping of the study area for Mid-term HTS Review 
The sampling criteria took into consideration facilities that were used initially, during the development of the strategy, on account of being high volume facilities with optimal service mix. The total number of facilities selected (36) are as tabulated in Table 1.

\begin{tabular}{|c|c|c|c|}
\hline Manicaland Province & Mash West Province & Matabeleland South Province & Midlands Province \\
\hline Mutare District & Chegutu District & Gwanda District & Gweru District \\
\hline Mutare Provincial Hospital & Katanga Utano Clinic & Gwanda Provincial Hospital & Gweru Provincial Hospital \\
\hline Zimunya Clinic & Pfupajena Municipal Clinic & Phakama Clinic & Chikwingwizha Mission Hospital \\
\hline Mt Zuma Clinic & Selous Clinic & Manama Mission Hospital & Lower Gweru Clinic \\
\hline Chipinge District & Makonde District & Mangwe District & Kwekwe District \\
\hline Chikore Mission Hospital & Chinhoyi Provincial Hospital & Plumtree District Hospital & Kwekwe General Hospital \\
\hline Chipinge Town Clinic & Chikonohono Municipal Clinic & Tshitshi Clinic & Amaveni Clinic \\
\hline Tanganda Rural Health Centre & Alaska Municipal Clinic & Dingumuzi Clinic & Nyoni Rural Health Centre \\
\hline Makoni District & Sanyati District & Umzingwane District & Zvishavane District \\
\hline Rusape District Hospital & Kadoma General Hospital & Nhlangano Clinic & Mandava Health Centre \\
\hline Katsenga Rural Health Centre & Ordoff Clinic & Nswazi Clinic & Mabasa Clinic \\
\hline
\end{tabular}

Table 1: Health Facilities for HTS Mid-term Evaluation, 2019

\section{Data Variables, Sources of Data and Data Collection}

Routinely collected program data (October to December 2018) were extracted from facility HTS registers using a structured data collection form. Data for a similar period and facilities entered into the national electronic database (DHIS2) was reviewed to triangulate extracted data and assess completeness and accuracy. Qualitative data was collected through key-informant interviews conducted across all levels of service provision to acquire an in-depth appreciation of program implementation dynamics. Data collection tools were pre-tested at four Harare City Health Department clinics in Kuwadzana, Budiriro, Marlborough and Rujeko and adjusted for clarity of questions.

\section{Analysis and Statistics}

Quantitative data collected from the assessment were extracted from the paper-based tally sheets and analyzed using excels to generate frequencies and proportion. Stata version 12 was used for crude and adjusted odds ratios. Quantitative data analysis was conducted on the Survey CTO platform and Atlas and organized according to the HTS strategy thematic areas.

\section{Ethics Approval}

Exemption from ethical approval bodies was obtained from the Ministry of Health Institutional Ethical Review Board (IRB), considering that the data utilised is routinely collected for programming and the interviews conducted were part of routine Support and Supervision activities, which are a component of programming. Written informed consent was obtained from key informants.

\section{Results}

\section{Demographic Characteristics}

\section{Baseline Characteristics}

Of 34,605 patients, females constituted $70 \%(n=24,221)$ and $36.5 \%(n=12,615)$ patients belonged to the age-group $25-49$ years. Most, $32.7 \%(n=11,300)$ of the tests done were for PMTCT, encompassing ANC, Labor and Delivery and Post Delivery testing. Provider-initiated testing accounted for $25.8 \%(n=8,938)$ of the total tests done, whilst Confirming a reactive Self-test result accounted for the least, $0.1 \%(n=13)$ number of tests done. Among the clients tested for HIV, 67.2\% $(n=23,265)$ were retests. Across the districts, Gweru recorded the highest, $13.2 \%(n=4,575)$ number of tests done whilst Umzingwane recorded the least, $2.1 \%(n=744)$ number of tests done (Table 2$)$.

\begin{tabular}{|c|c|c|}
\hline \multicolumn{1}{|c|}{ Variable } & Number & $(\%)^{*}$ \\
\hline Total & $\mathbf{3 4 , 6 0 5}$ & $\mathbf{- 1 0 0}$ \\
\hline Age in years & & \\
\hline o $<5$ & 1,093 & $(3.2)$ \\
\hline o 9-May & 461 & $(1.3)$ \\
\hline o 14-Oct & 1,206 & $(3.5)$ \\
\hline o $15-19$ & 3,524 & $(10.2)$ \\
\hline o 20-24 & 6,588 & $(19.0)$ \\
\hline
\end{tabular}




\begin{tabular}{|c|c|c|}
\hline Variable & Number & $(\%)^{*}$ \\
\hline о $25-49$ & 12,615 & (36.5) \\
\hline $0>/=50$ & 5,684 & (16.4) \\
\hline o Not Documented & 3,434 & $(9.9)$ \\
\hline \multicolumn{3}{|l|}{ Gender } \\
\hline o Male & 10,384 & $(30.0)$ \\
\hline o Female & 24,221 & $(70.0)$ \\
\hline \multicolumn{3}{|l|}{ Reason for HIV Testing } \\
\hline o Confirming Self-test & 13 & $(0.1)$ \\
\hline o Index Contact & 1,752 & $(5.1)$ \\
\hline o Exposed Infant & 701 & $(2.0)$ \\
\hline о РMTCT & 11,300 & $(32.7)$ \\
\hline o STI & 819 & $(2.4)$ \\
\hline o VMMC & 1,459 & $(4.2)$ \\
\hline o Client Initiated & 7,525 & (21.7) \\
\hline o Provider Initiated & 8,938 & (25.8) \\
\hline o TB Patient & 362 & $(1.0)$ \\
\hline o Sexual Abuse & 474 & $(1.4)$ \\
\hline o PrEP & 40 & $(0.1)$ \\
\hline o Family Planning & 419 & $(1.2)$ \\
\hline o Other & 763 & $(2.2)$ \\
\hline \multicolumn{3}{|l|}{ Type of HIV Test } \\
\hline o First Test & 7,906 & $(22.8)$ \\
\hline o Retest & 23,265 & $(67.2)$ \\
\hline o Not Documented & 3,434 & (9.9) \\
\hline \multicolumn{3}{|l|}{ HIV Test Result } \\
\hline o Negative & 32,878 & $(95.0)$ \\
\hline o Positive & 1,727 & $(5.0)$ \\
\hline \multicolumn{3}{|l|}{ Testing District } \\
\hline o Mangwe & 2040 & $(5.9)$ \\
\hline o Sanyati & 3232 & $(9.3)$ \\
\hline o Gwanda & 2239 & $(6.5)$ \\
\hline o Chipinge & 2350 & $(6.8)$ \\
\hline o Mutare & 2201 & $(6.4)$ \\
\hline o Gweru & 4575 & $(13.2)$ \\
\hline o Kwekwe & 3443 & $(9.9)$ \\
\hline o Chegutu & 4279 & $(12.4)$ \\
\hline o Makonde & 3997 & (11.6) \\
\hline o Umzingwane & 744 & $(2.1)$ \\
\hline o Makoni & 3424 & $(9.9)$ \\
\hline o Zvishavane & 2081 & $(6.0)$ \\
\hline
\end{tabular}

${ }^{*}$ Column percentage

STI - Sexually Transmitted Infections

VMMC - Voluntary Medical Male Circumcision

TB - Tuberculosis

PrEP - Pre-exposure Prophylaxis

PMTCT - Prevention of Mother to Child Transmission; includes Antenatal, Labor and Delivery and Post-Delivery testing

Index Testing include Partner testing

Provider Initiated Testing encompass other register variables; Occupational, Nutrition, Ongoing risk and Diagnosis

Table 2: Clinical and demographic profile of clients who tested for HIV in for 36 Health Facilities, October to December, Zimbabwe, 2018. $(\mathrm{N}=34,605)$ 
All visited facilities were implementing the full complement of mainstream HIV testing services that include Provider Initiated Testing and Counselling (PITC), Client Initiated Testing and Counseling (CITC), Index Contact Tracing and Testing both at the facility and in the community. HIVST was only available at $1 / 3$ of the visited facilities influenced by partner support that does not cover the entire geographical landscape of the country. Satisfactory $(80 \%, 462 / 576)$ program knowledge levels on policy direction, guiding documents, such as the Operational Service Delivery Manual (OSDM), the application of the screening tool to determine eligibility for testing, as well as counselling skills for varied post-test scenarios, was recorded.

\section{HIV Positivity}

Overall, 6.1\% ( $\mathrm{N}=1,908)$ of clients tested for HIV obtained a positive result. The 25-49-year age group recorded the highest positivity at $8.4 \%(n=1,058)$, whilst the 10 -14-year old recorded the least positivity at $1.8 \%(n=22)$. In adjusted analysis, the probability of obtaining a positive HIV diagnosis was highest in the 25-49 age category (aOR=2.77 [95\%CI, 1.97-3.9]), followed by $>/=50$ age group $(\mathrm{aOR}=1.83$ [95\%CI, 1.29-2.61]). Males recorded 6.9\% $(\mathrm{n}=680 / 9,902)$ positivity in comparison with females at 5.8\% $(\mathrm{n}=1,228 / 21,269)$. Adjusted for age and reason for testing, males were 20\% (95\%CI, 1.09-1.33) more likely to obtain a positive HIV test results compared with females.

Disaggregated by reason for testing, Index contact testing recorded 13.5\% ( $n=236 / 1,752)$ positivity, followed by Exposed infants at 7\% (49/701). In adjusted analysis, Index contacts were 4.97 times more likely to obtain a positive HIV diagnosis (95\%CI, 2.689.19), followed by exposed infants ( $\mathrm{aOR}=2.40$ [95\%CI, 1.23-4.67]). There was no positive diagnosis among clients who tested in order to access and while on Pre-exposure prophylaxis. Of 7,906 first-time testers, $8.1 \%$ (643) obtained an HIV positive diagnosis, whilst 23,265 retests recorded 5.4\% ( $\mathrm{n}=1,256)$ positivity. Among districts, Mangwe recorded 8.8\% (176/2004) positivity, followed by Gwanda at $8.2 \%(\mathrm{n}=162 / 1,983)$ positivity. Umzingwane recorded a positivity of $4.6 \%(34 / 737)$ in comparison with Makoni at $4.3 \%(148 / 3,472)$ (Table 3).

\begin{tabular}{|c|c|c|c|c|c|}
\hline \multirow{2}{*}{ Variable } & \multirow{2}{*}{$\begin{array}{c}\text { Total } \\
\mathbf{N}\end{array}$} & \multicolumn{2}{|c|}{ HIV Positive ${ }^{*}$} & \multirow{2}{*}{ OR (95 CI) } & \multirow{2}{*}{$\mathrm{aOR}(95 \mathrm{CI})^{*}$} \\
\hline & & $\mathbf{N}$ & $(\%)^{* *}$ & & \\
\hline Total & 31,171 & 1,908 & $(6.1)$ & - & - \\
\hline \multicolumn{6}{|l|}{ Age in years } \\
\hline $0<5$ & 1,093 & 35 & $(3.2)$ & Ref & Ref \\
\hline o 9-May & 461 & 20 & $(4.3)$ & $0.73(0.42,1.28)$ & $1.37(0.78,2.40)$ \\
\hline o 14 -Oct & 1,206 & 22 & $(1.8)$ & $1.79(1.04,3.06)$ & $0.56(0.33,0.96)^{\wedge}$ \\
\hline o $15-19$ & 3,524 & 105 & $(3.0)$ & $1.08(0.73,1.59)$ & $0.93(0.63,1.37)$ \\
\hline о $20-24$ & 6,588 & 343 & $(5.2)$ & $0.60(0.42,0.86)$ & $1.66(1.17,2.37)^{\wedge}$ \\
\hline о $25-49$ & 12,615 & 1,058 & $(8.4)$ & $0.36(0.26,0.51)$ & $2.77(1.97,3.90)^{\wedge}$ \\
\hline $\mathrm{o}>/=50$ & 5,684 & 325 & $(5.7)$ & $0.55(0.38,0.78)$ & $1.83(1.29,2.61)$ \\
\hline \multicolumn{6}{|l|}{ Gender } \\
\hline o Male & 9,902 & 680 & $(6.9)$ & $1.20(1.09,1.33)$ & $1.20(1.09,1.33)^{\wedge}$ \\
\hline o Female & 21,269 & 1,228 & $(5.8)$ & Ref & Ref \\
\hline \multicolumn{6}{|c|}{ Reason for HIV Testing* } \\
\hline o Index Contact & 1,752 & 236 & $(13.5)$ & $4.97(2.68,9.19)$ & $4.97(2.68,9.19)^{\wedge}$ \\
\hline o Exposed Infant & 701 & 49 & $(7.0)$ & $2.40(1.23,4.67)$ & $2.40(1.23,4.67)^{\wedge}$ \\
\hline о РMTCT & 11,300 & 378 & $(3.3)$ & $1.10(0.60,2.03)$ & $1.10(0.60,2.03)$ \\
\hline o STI & 819 & 45 & $(5.5)$ & $1.86(0.95,3.63)$ & $1.86(0.95,3.63)$ \\
\hline o VMMC $C^{* * *}$ & 1,459 & 4 & $(0.3)$ & $0.09(0.02,0.30)$ & $0.88(0.03,0.28)^{\wedge}$ \\
\hline o Client Initiated & 7,525 & 365 & $(4.9)$ & $1.63(0.88,2.99)$ & $1.63(0.88,2.99)$ \\
\hline o Provider Initiated & 8,975 & 606 & $(6.8)$ & $2.31(1.26,4.23)$ & $2.31(1.26,4.23)^{\wedge}$ \\
\hline o TB Patient & 362 & 11 & $(3.0)$ & Ref & Ref \\
\hline o Sexual Abuse ${ }^{* * *}$ & 474 & 4 & $(0.8)$ & $0.27(0.06,0.93)$ & $0.27(0.09,0.86)^{\wedge}$ \\
\hline o PrEP $P^{* * *}$ & 40 & 1 & $(0)$ & $0.82(0.02,5.91)$ & $0.82(0.02,5.91)$ \\
\hline o Family Planning & 419 & 10 & $(2.4)$ & $0.78(0.33,1.86)$ & $0.78(0.33,1.86)$ \\
\hline o Other ${ }^{* * *}$ & 763 & 6 & $(0.8)$ & $0.25(0.08,0.75)$ & $0.25(0.09,0.69)^{\wedge}$ \\
\hline \multicolumn{6}{|l|}{ Type of HIV Test } \\
\hline o First Test & 7,906 & 643 & $(8.1)$ & $1.55(1.41,1.71)$ & $1.56(1.41,1.71)^{\wedge}$ \\
\hline o Retest & 23,265 & 1,256 & $(5.4)$ & Ref & Ref \\
\hline Testing District & & & & & \\
\hline
\end{tabular}




\begin{tabular}{|l|c|c|c|c|c|}
\hline \multirow{2}{*}{ Variable } & Total & \multicolumn{2}{|c|}{ HIV Positive } & \multirow{2}{*}{ OR (95 CI) } & \multirow{2}{*}{ aOR $(\mathbf{9 5} \text { CI })^{*}$} \\
\cline { 2 - 4 } & $\mathbf{N}$ & $\mathbf{N}$ & $\mathbf{( \% )}^{* *}$ & & - \\
\hline Total & $\mathbf{3 1 , 1 7 1}$ & $\mathbf{1 , 9 0 8}$ & $\mathbf{( 6 . 1 )}$ & - & - \\
\hline o Mangwe & 2,004 & 176 & $(8.8)$ & $2.24(1.68,2.99)$ & $2.24(1.68,2.99)^{\wedge}$ \\
\hline o Sanyati & 2,874 & 210 & $(7.3)$ & $1.83(1.38,2.43)$ & $1.83(1.38,2.43)^{\wedge}$ \\
\hline o Gwanda & 1,983 & 162 & $(8.2)$ & $2.07(1.55,2.77)$ & $2.07(1.55,2.77)^{\wedge}$ \\
\hline o Chipinge & 1,626 & 67 & $(4.1)$ & Ref & Ref \\
\hline o Mutare & 2,009 & 128 & $(6.4)$ & $1.58(1.17,2.14)$ & $1.58(1.17,2.14)^{\wedge}$ \\
\hline o Gweru & 4,358 & 291 & $(6.7)$ & $1.67(1.27,2.19)$ & $1.67(1.27,2.19)^{\wedge}$ \\
\hline o Kwekwe & 3,028 & 152 & $(5.0)$ & $1.23(0.92,1.65)$ & $1.23(0.92,1.65)$ \\
\hline o Chegutu & 3,702 & 234 & $(6.3)$ & $1.57(1.19,2.07)$ & $1.57(1.19,2.07)^{\wedge}$ \\
\hline o Makonde & 3,586 & 188 & $(5.2)$ & $1.29(0.97,1.71)$ & $1.29(0.97,1.71)$ \\
\hline o Umzingwane & 737 & 34 & $(4.6)$ & $1.13(0.74,1.72)$ & $1.13(0.74,1.72)$ \\
\hline o Makoni & 3,472 & 148 & $(4.3)$ & $1.04(0.77,1.39)$ & $1.04(0.77,1.39)$ \\
\hline o Zvishavane & 1,792 & 118 & $(6.6)$ & $1.64(1.21,2.23)$ & $1.64(1.21,2.23)$ \\
\hline
\end{tabular}

OR - Odds Ratio; aOR - adjusted Odds Ratio; CI - confidence interval

${ }^{*}$ Confirming Self-test was excluded from the model as a reason for HIV testing

\# Positivity excludes missing variables

${ }^{*}$ Row percentages; \#Modified Poisson regression for aOR; ${ }^{\wedge} \mathrm{p}<0.05$, ${ }^{* * *}$ Fisher's Exact

STI - Sexually Transmitted Infections

VMMC - Voluntary Medical Male Circumcision

TB - Tuberculosis

PrEP - Pre-exposure Prophylaxis

PMTCT - Prevention of Mother to Child Transmission

Table 3: Clinical and demographic profile of clients who tested for HIV in for 36 Health

Facilities, October to December, Zimbabwe, 2018. $(\mathrm{N}=34,605)$

\section{HIV Testing Services Cascade}

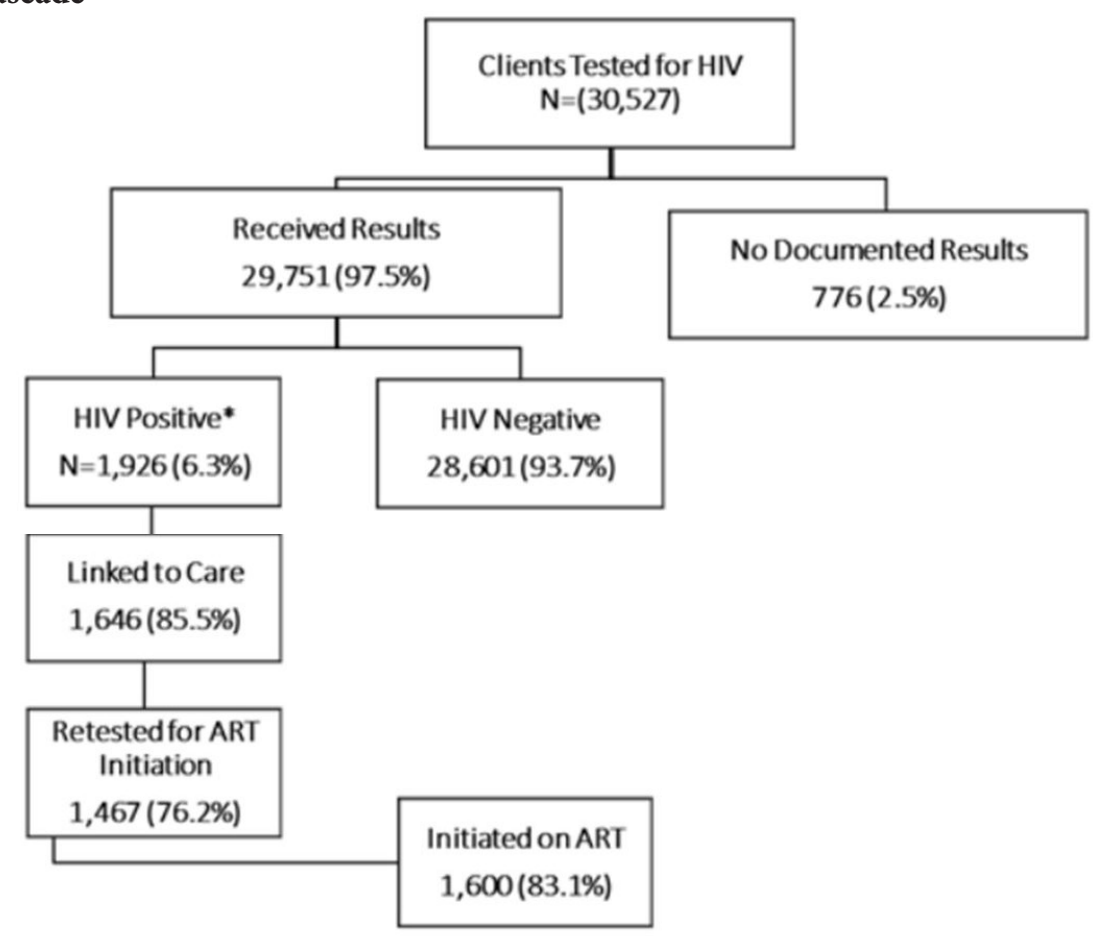

*Total number HIV positive becomes the denominator ART-Antiretroviral Therapy

Figure 3: HIV Testing Services Cascade Extracted from District Health Information System (DHIS2) for 36 Health Facilities, October to December, Zimbabwe, 2018. (N=30,527) 
An 11.8\% (30,527/ 34,605) discrepancy was observed during data triangulation of the abstracted data with monthly summaries and data entered into the national electronic District Health Information System database (DHIS2), with fewer entries appearing in the electronic system. The abstracted data was used to generate cascades (Figure 3).

Of 30,527 clients tested for HIV, 97\% ( $\mathrm{n}=29,751)$ had their results documented. Out of the total clients tested, 6.3\% ( $\mathrm{n}=1,926)$ obtained a HIV positive result. Among the HIV positive clients, $85.5 \%(n=1,646)$ were linked to care and $76.2 \%(n=1,467)$ were retested for ART initiation. A total of $83.1 \%(n=1,600)$ were initiated on ART indicating an overall 6.9\% $(n=326)$ attrition from HIV diagnosis to treatment initiation (Figure 3).

\section{HIV Self-Testing Cascade}

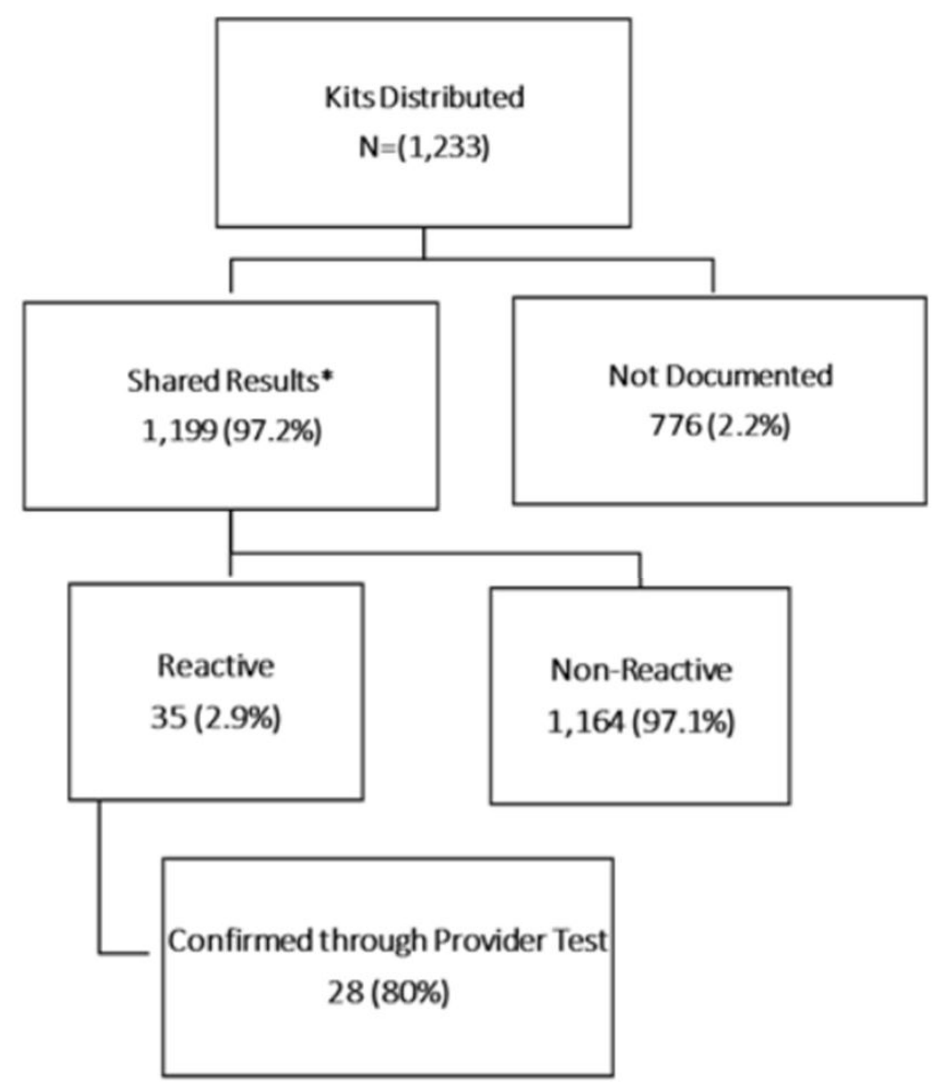

"Total number Shared Results becomes the denominator

Figure 4: HIV Self-Testing Cascade Extracted from District Health Information System (DHIS2) for 36 Health Facilities, October to December, Zimbabwe, 2018. (N=1,233)

Of 1,233 clients that obtained HIV Self-test kits, $97.2 \%(n=1,199)$ shared results as documented evidence of having utilised the given kits. Among the clients who shared results, 2.9\% $(n=35)$ reported reactive Self-test results and of these, $80 \%(n=28)$ were confirmed using provider delivered testing as depicted in Figure 4.

\section{Discussion}

A key finding in this study is that targeted HIV testing, through index contact tracing resulted in high positivity and effective utilization of HIV test-kits. The use of a screening tool to assess eligibility for testing resulted in a reduced number of tests but positivity maintained a decreasing trajectory.

\section{Strengths}

Availability of primary source documents, such as HTS registers at all visited facilities facilitated the data abstraction process. In addition, the sampled 36 health facilities provided a large sample size that enabled us to draw inferences on the population of the country. The large sample also facilitated an in-depth evaluation of the current state of program implementation and progress to date towards the achievement of the set target to achieve $90 \%$ of people living with HIV being identified by 2020. Policy documents that guide health workers to fully implement the HTS strategy were available at most testing sites, including testing algorithms. The study was done using routinely collected programmatic data which was representative of the reality on the ground. Most health workers were conscious of the HTS policy direction focusing on targeted testing rather than testing for coverage, though this did not translate to implementation fidelity. 


\section{Limitations}

Discrepancies between data abstracted from HTS registers, monthly summaries and DHIS2 during data triangulation exposed data entry or computation errors that could be rectified by onsite data analysis, and cascade generation, which was not in practice at most facilities. Reporting HIVST data through templates parallel to routine reporting structures impeded comprehensive data reporting and triangulation, resulting in reliance on data entered into registers only. The implementation of HIV Self-testing was limited to partner supported sites who provide the test kits.

\section{Interpretation of Key Findings}

This study provided important insights into the performance of the HTS Programme in Zimbabwe. First, index contacts were nearly 5 times more likely to test HIV positive, with the highest positivity of $13.5 \%$, regardless of recording one of the least numbers of HIV tests conducted $(5 \% ; n=1,752 / 34,605)$. This finding is consistent with previous studies that vindicate index contact testing for effective utilization of resources to identify people living with HIV, with a yield of ranging from $35-62 \%$ [13,14]. Secondly, the overall number of clients who tested for the first time in their entire lives was less when compared with repeat tests, and yet recorded a higher positivity rate of $8.1 \%$, against repeat tests at $5.4 \%$, whilst the probability of testing HIV positive being as high as $56 \%$ for first tests. This finding suggests that there may be an unmet need for HIV testing services, particularly for specific subgroups of the population or low acceptability of HIV testing, resulting in low testing levels [13]. This is particularly so when the age of the participants in brought into context, which is predominantly the 25-45-year-olds, with other participants being above 65 years of age. High positivity for first-time testers is contrary to what has been reported globally [14].

Third, the high number of clients who retested for HIV, 67.2\% ( $n=23,265)$, and yet recording low positivity may be suggestive of "over-testing" or high-risk perception. Clients who obtain a negative HIV diagnosis are retested yearly if they fall into the general population category and retested 3 monthly if they are at ongoing risk for HIV transmissions, such as discordant relationships and those on PrEP, according to the screening tool [15]. The retests were mostly contributed by the high HIV testing frequency in pregnancy, $32.7 \%(n=11,300)$, which has a varied algorithm that culminates in a woman obtaining up to 7 HIV tests from booking visit until the child is 9 months post-delivery. Fourth, HIV Self-testing contributed $<1 \%$ of the total tests done, despite being part of the innovations aimed to facilitate the expansion of access to HIV testing services through self-screen for clients who do not readily access or accept provider offered testing services.

This finding is against the background of HIVST being highly acceptable among the Zimbabwean population [16]. The limited geographical coverage of the program, as a consequence of dependence on partner support, coupled with subdued commodity supply, maybe hindering the innovation's potential to decongest high volume facilities, improve health worker efficiency by reducing workload, as well as unveiling HIV testing services to rejectors and hard to reach population through facility and community distribution models [15]. The nearly $12 \%$ discrepancy between data extracted from primary sources at facilities and DHIS2 indicate that as much as 4,078 documented HIV tests had not been entered into the electronic database (DHIS2). Documentation errors exposed during triangulation of facility registers, Monthly summaries and the electronic database (dhis2) suggest that this may have been contributed by the following;

a. Some of the monthly summaries were not entered into DHIS2 or may have been partly entered,

b. Data transcription errors whereby the information entered into DHIS2 is not a true reflection of what is on the paper-based, source and summary documents (Heale and Forbes 2013).

Finally, cascade generation using the electronic datasets exposed leakages at every step, with the overall attrition being 6.9\% ( $\mathrm{n}=326)$, from HIV diagnosis to treatment initiation. The largest attrition was $23.8 \% \%(n=1,467)$ leakage from testing positive to retesting for ART initiation. This finding is consistent with findings by previous studies which indicate that $80-90 \%$ of HIV identified persons are initiated on treatment [17-19]. Clients who are diagnosed and yet do not initiate treatment present the risk of onward transmission of the virus, negatively impacting epidemic control efforts. The HIVST cascade indicated that as high as $97.2 \%(n=1,199)$ of selftesters shared results, whilst positivity was $2.9 \%$, which is lower than what was previously obtained [16]. Only $80 \%$ of positive selftesters were further tested to confirm their results, consistent with the program standard operating practice [20]. Clients who selftest positive and are not confirmatory tested by the provider miss the opportunity of early treatment, a critical element in reducing HIV morbidity and onward transmission of the virus. Post-test linkage channels and available services should be emphasized to clients at the point of kit distribution [16]. Notably, cascade generation and onsite data analysis, which facilitate data ownership and utilisation at local level were not being practised.

\section{Implications for Policy and Practice}

Targeted tested is the mainstay of HTS programming to achieve epidemic control. This requires strengthening to ensure that all identified HIV positives, as well as high viral load clients, are offered index testing, have their contacts elicited at 1.1.5-2 as recommended and ensure that all elicited contacts are tested through facility and community initiatives. This measure will improve yields and facilitate epidemic control. Routine, onsite data analysis through cascade generation will facilitate identification of areas of weakness and enable redress. Entry point and overall institutional cascades enable timely feedback that can facilitate immediate corrective measurement. Regular data meetings, that include partner seconded personnel is an important platform for data triangulation and analysis for consistency and problem-solving. 
Increasing HIVST kit distribution, particularly to pregnant women as secondary distribution to their partners. This approach has been demonstrated to enhance HTS through the following; for negative testers at the facility, self-test promotes couple testing and case finding in view of seroconversions that occur during subsequent tests during pregnancy and lactation [16], positive testers can leverage on HIVST to disclose to their partners as well as contact tracing [20].

\section{Future Research}

First, a qualitative study to explore the most feasible and acceptable way to offer index contact tracing in view of the low elicitation rate, which may be contributed by the sensitive nature of disclosing sexual contacts, particularly those that are not socially acceptable and outside of the expected relations. Second, operational research to summative evaluate the HTS strategy implementation, considering the recommendations proffered during the mid-term evaluation. There is also a need for similar studies, utilizing routinely collected data (enabling assessment of trends) in other parts of Zimbabwe [21-25].

\section{Conclusion}

Our findings on the formative evaluation of the HTS strategy reflected a need to enhance implementation fidelity, particularly on targeted testing, promoting index contact tracing in order to meet the strategic objectives set for summative evaluation at the end of 2020. We identified data discrepancy that could be addressed by onsite cascade generation, triangulation and analysis, to enable consistent data reporting.

\section{Acknowledgement}

Special gratitude goes to the entire HTS team, the AIDS and TB Unit Director, Dr Mugurungi and Clinton Health Access Initiative (CHAI) staff for the support and hard work conducting the midterm data collection exercise. I also express my gratitude to the entire staff at the health facilities where the data collection exercise was conducted for the assistance they rendered during the entire study process. This operational research was conducted as an initiative to utilize collected programme data for scientific writing.

\section{References}

1. Zimbabwe Ministry of Health and Child Care, National AIDS Council, Zimbabwe (2017) HIV Estimates Report.

2. Ministry of Health and Child Care (MoHCC), Zimbabwe (2017) National HIV Testing Services Strategy, 2017-2020.

3. World Population Review (2019) Zimbabwe Population 2019 (Demographics, Maps, Graphs).

4. Zimbabwe Ministry of Health and Child Care (2005) Zimbabwe National Guidelines on Hiv Testing and Counselling.

5. UNDP and Government of Zimbabwe (2017) Zimbabwe Human Development Report 2017. Climate Change and Human Development: Towards Building a Climate Resilient Nation. Harare, Zimbabwe.

6. Unaids (2020) 90-90-90: An ambitious treatment target to help end the AIDS epidemic.

7. Moyo Sikhulile, Eduan Wilkinson, Vladimir Novitsky, Alain Vandormael, Simani Gaseitsiwe, et al. (2015) Identifying recent HIV infections: From serological assays to genomics. Viruses 7: 5508-24.

8. Nah Kyeongah, Hiroshi Nishiura, Naho Tsuchiya, Xiaodan Sun, Yusuke Asai, et al. (2017) Test-and-treat approach to HIV/AIDS: a primer for mathematical modeling. Theor Biol \& Med Model 14: 16.

9. Zimbabwe Ministry of Health and Child Care, National AIDS Council, Zimbabwe (2018) Global Aids Response Progress Report 2018 Gam Zimbabwe Country Report.

10. Zimbabwe Ministry of Health and Child Care (2018) Ministry of Health and Child Welfare, National Tuberculosis Program -Strategic Plan (2017-2020). Harare, Zimbabwe.

11. Zimstat (2012) Zimbabwe Population Census 2012. Population Census Office pp. 1-151.

12. Zimbabwe Country Profile (2019) UN in Zimbabwe.

13. Ahmed Saeed, Rachael A Sabelli, Katie Simon, Nora E Rosenberg, Elijah Kavuta, et al. (2017) Index case finding facilitates identification and linkage to care of children and young persons living with HIV/AIDS in Malawi. Trop Med Int Health 22: 1021-9.

14. Management Health Sciences (MHS) (2018) Index Case Testing: A Promising Strategy for Achieving Hiv Epidemic Control Background.

15. Opt-home (2017) Counselling messages - Choosing Clinic-Based or Home-Based HIV Testing.

16. Ingold Heather, Ombeni Mwerinde, Anna Laura Ross, Ross Leach, Elizabeth L Corbett, et al. (2019) The Self-Testing AfRica (STAR) Initiative: accelerating global access and scale-up of HIV self-testing. J Int AIDS Soc 22: e25249.

17. Fox Matthew P (2016) Are we shifting attrition downstream in the HIV cascade? The Lancet HIV 3: e554-e555.

18. Ruadze Ekaterine, Nikoloz Chkhartishvili, Otar Chokoshvili, Tengiz Tsertsvadze (2017) Cascade of care among HIV patients diagnosed in 2013 in Georgia: Risk factors for late diagnosis and attrition from HIV care. SAGE Open Medicine 5: 205031211773197.

19. Kerkerian Genevieve, Mary Kestler, Allison Carter, Lu Wang, Nadine Kronfli, et al. (2018) Attrition across the HIV cascade of care among a diverse cohort of women living with HIV in Canada. J Acquired Immune Deficiency Syndromes 79: 226-36.

20. World Health Organization (WHO) (2016) Guidelines on Hiv Self-Testing and Partner Notification Supplement to Consolidated Guidelines on Hiv Testing Services Annex 32. Report on the values and preferences on HIV self-testing and partner notification in Indonesia.

21. Heale Roberta, Dorothy Forbes (2013) Understanding triangulation in research. Evidence-Based Nursing 16: 98. 
22. Ministry of Health and Child Care (MoHCC), Zimbabwe (2016) The U.S. President's Emergency Plan for AIDS Relief (PEPFAR), The U.S. Centers for Disease Control and Prevention (CDC), Zimbabwe National Statistics Agency (ZIMSTAT), The Biomedical Research and Training Institute (BRTI), Lancet Laboratories, Statistical Center for HIV/AIDS Research and Prevention (SCHARP), Westat, and ICAP at Columbia University. ZIMBABWE POPULATION-BASED HIV IMPACT ASSESSMENT ZIMPHIA 2015-2016.

23. USAID (201) Partner and Family-Based Index Case Testing: A Standard Operating Procedure (SOP).

24. World Population Review (2018) Zimbabwe Population.

25. Zimbabwe Ministry of Health and Child Care (2016) National AIDS Council, Zimbabwe. Global Aids Response Progress Report 2016. 